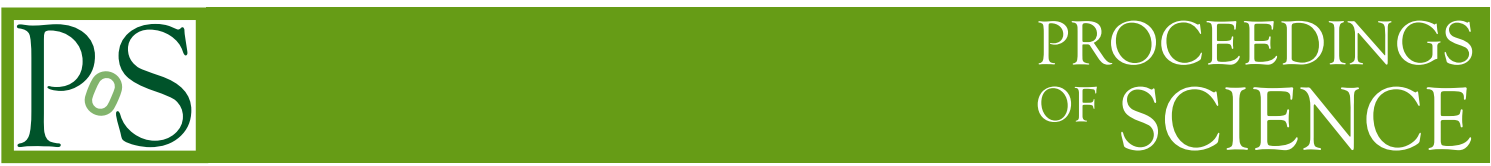

\title{
Probing $p_{\mathrm{T}}$-dependent flow vector fluctuations with ALICE
}

\author{
Emil Gorm Nielsen for the ALICE collaboration ${ }^{a, *}$ \\ ${ }^{a}$ Niels Bohr Institute, \\ Blegdamsvej 17, Copenhagen, Denmark \\ E-mail: emil.gorm.nielsen@cern.ch
}

\begin{abstract}
One of the main goals of ultra-relativistic nuclear collisions is to create a new state of matter called the quark-gluon plasma (QGP) and study its properties. One of the experimental observables is the anisotropic flow $v_{n}$, defined as the correlation of the azimuthal angle of each particle with respect to a common symmetry plane $\Psi_{n}$. The $v_{n}$ and $\Psi_{n}$ represent the magnitude and the phase of a complex flow vector $V_{n}$, respectively. Azimuthal anisotropies are traditionally measured using 2- and/or multi-particle correlations over a large range in $p_{\mathrm{T}}$ and $\eta$. However, hydrodynamic calculations show that the event-by-event fluctuations in the initial conditions and the dynamics during the system expansion lead to flow vector fluctuation in $p_{\mathrm{T}}$ and/or $\eta$ (also called de-correlations of flow vector), including flow magnitude and flow angle fluctuations.

In this proceeding, we present the evidence of $p_{\mathrm{T}}$-dependent flow vector fluctuations in $\mathrm{Pb}-\mathrm{Pb}$ collisions at $\sqrt{s_{\mathrm{NN}}}=5.02 \mathrm{TeV}$, using both $v_{n}\{2\} / v_{n}$ [2] and $r_{n}$ observables. In addition, newly proposed four-particle correlations are used to study the contributions of flow magnitude and flow angle fluctuations separately. Considering that the size of flow vector fluctuations is sensitive to both initial conditions and the properties of the created QGP, these measurements will help us better constrain hydrodynamic models.
\end{abstract}

The Eighth Annual Conference on Large Hadron Collider Physics-LHCP2020

25-30 May, 2020

online

${ }^{*}$ Speaker 


\section{Introduction}

In heavy-ion collisions, hot and dense matter is created. This matter is commonly called the quark-gluon plasma (QGP). As the QGP expands, anisotropies in the initial geometry are transferred into final state momentum-space correlations by the hydrodynamic evolution of the medium. The anisotropic flow can be described by a Fourier expansion of the azimuthal distribution of the emitted particles [1]:

$$
\frac{\mathrm{d} N}{\mathrm{~d} \varphi}=1+2 \sum_{n=1}^{\infty} V_{n} e^{i n \varphi}
$$

with

$$
V_{n}=v_{n} e^{i n \Psi_{n}},
$$

where $V_{n}$ is the flow vector with magnitude $v_{n}$ and angle $\Psi_{n}$.

The flow vector may fluctuate as a function of transverse momentum $p_{\mathrm{T}}$, due to event-byevent fluctuations of the initial state and the dynamics of the medium during expansion. These $p_{\mathrm{T}}$-dependent flow vector fluctuations are measured with the ratio [2]:

$$
\frac{v_{n}\{2\}}{v_{n}[2]}=\frac{\left\langle v_{n}\left(p_{\mathrm{T}}^{a}\right) v_{n} \cos n\left[\Psi_{n}\left(p_{\mathrm{T}}^{a}\right)-\Psi_{n}\right]\right\rangle}{\sqrt{\left\langle v_{n}\left(p_{\mathrm{T}}^{a}\right)^{2}\right\rangle} \sqrt{\left\langle v_{n}^{2}\right\rangle}},
$$

or with the factorization ratio [3]:

$$
r_{n}=\frac{\left\langle v_{n}\left(p_{\mathrm{T}}^{a}\right) v_{n}\left(p_{\mathrm{T}}^{t}\right) \cos \left[n\left(\Psi_{n}\left(p_{\mathrm{T}}^{a}\right)-\Psi_{n}\left(p_{\mathrm{T}}^{t}\right)\right]\right\rangle\right.}{\sqrt{\left\langle v_{n}\left(p_{\mathrm{T}}^{a}\right)^{2}\right\rangle\left\langle v_{n}\left(p_{\mathrm{T}}^{t}\right)^{2}\right\rangle}} .
$$

Here the associate particle is selected with transverse momentum $p_{\mathrm{T}}^{a}$, and the trigger particle is selected with with transverse momentum $p_{\mathrm{T}}^{t}$. In both cases a value smaller than unity indicates the presence of $p_{\mathrm{T}}$-dependent flow vector fluctuations. The difference between $v_{n}\{2\} / v_{n}$ [2] and $r_{n}$ is that the former carries information from the differential and $p_{\mathrm{T}}$-integrated flow, while the latter looks at the detailed correlation structure at $p_{\mathrm{T}}^{a}$ and $p_{\mathrm{T}}^{t}$. Understanding the $p_{\mathrm{T}}$-dependent flow vector fluctuations will help us understand the dynamical expansion of the created medium and can further constrain initial conditions and QGP properties.

\section{Analysis method}

The analysis was performed on data from $\mathrm{Pb}-\mathrm{Pb}$ collisions at $\sqrt{s_{\mathrm{NN}}}=5.02 \mathrm{TeV}$ collected with the ALICE experiment [4] in 2015. A minimum bias trigger was used to select suitable events for analysis. The events are required to have a primary z-vertex less than $10 \mathrm{~cm}$ from the nominal interaction point. Tracks are selected from pseudorapidity $-0.8<\eta<0.8$ and in the kinematic region $0.2<p_{\mathrm{T}}<5.0 \mathrm{GeV} / c$. The particle correlations are calculated with the Generic Framework [5], which also corrects for non-uniformities in the detector acceptance. Non-flow effects due to correlations of particles from processes such as resonance decay or within jets are suppressed using the subevent method. The detector region is split into two subevents with a spatial separation between the subevents enforced by a gap in pseudorapidity, $|\Delta \eta|>0.8$. 


\section{Results}

Figure 1 shows the ratio $v_{2}\{2\} / v_{2}[2]$ as a function of $p_{\mathrm{T}}$ in different centrality classes. A clear deviation up to $15 \%$ from unity is observed in the $0-5 \%$ and $5-10 \%$ central collisions and it is observed that the deviation increases as $p_{\mathrm{T}}$ increases. The measurements are compared to the iEBE-VISHNU hydrodynamical model [6] with different initial conditions and parameters for the QGP properties. The first has AMPT initial conditions and $\eta / s=0.08$ [7], and the other has TRENTo initial conditions and temperature dependent $\eta / s(T)$ and $\zeta / s(T)$ [7]. The hydrodynamic calculations are consistent with the data for both models.

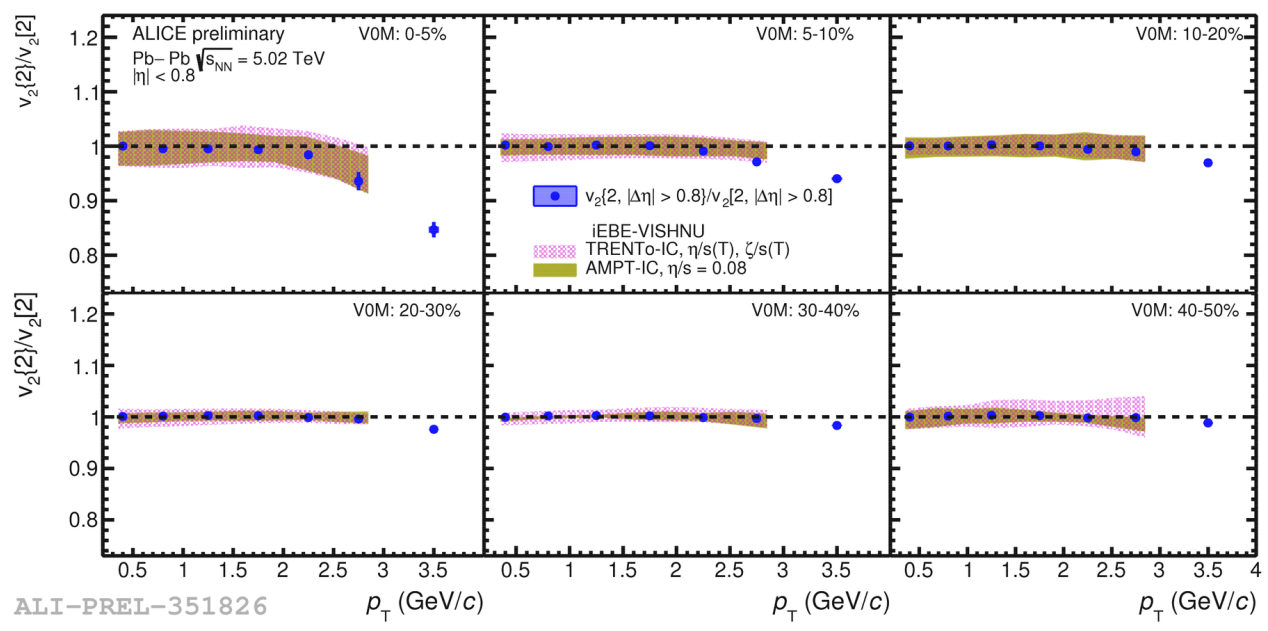

Figure 1: The ratio $v_{2}\{2\} / v_{2}$ [2] in $\mathrm{Pb}-\mathrm{Pb}$ collisions at $\sqrt{s_{\mathrm{NN}}}=5.02 \mathrm{TeV}$. The error bars correspond to statistical uncertainties and coloured boxes correspond to systematic uncertainties. Comparison with iEBEVISHNU hydrodynamic model [6] with TRENTo initial conditions and temperature dependent $\eta / s(T)$ and $\zeta / s(T)$ [7], and with AMPT initial conditions and $\eta / s=0.08$ [7] are shown in coloured bands.

Figure 2 shows the factorization ratio $r_{2}$ as a function of $p_{\mathrm{T}}^{a}$ with $0.2<p_{\mathrm{T}}^{t}<0.6$ in different centrality classes. A deviation of $r_{2}$ from unity up to $\sim 14 \%$ is observed for central collisions at high $p_{\mathrm{T}}$. The hydrodynamic calculations slightly underestimate the deviations, however, the model with AMPT initial conditions and $\eta / s=0.08$ describes the data marginally better.

A new observable is proposed to disentangle the contributions of flow magnitude and flow angle fluctuations:

$$
\begin{aligned}
C\left(\Psi_{n}^{a}, \Psi_{n}^{t}\right) \equiv \frac{\left\langle\left\langle\cos \left[n\left(\varphi_{1}^{a}+\varphi_{2}^{a}-\varphi_{3}^{t}-\varphi_{4}^{t}\right)\right]\right\rangle\right\rangle}{\left\langle\left\langle\cos \left[n\left(\varphi_{1}^{a}+\varphi_{2}^{t}-\varphi_{3}^{a}-\varphi_{4}^{t}\right)\right]\right\rangle\right\rangle} & =\frac{\left\langle v_{n}\left(p_{\mathrm{T}}^{a}\right)^{2} v_{n}\left(p_{\mathrm{T}}^{t}\right)^{2} \cos 2 n\left[\Psi_{n}\left(p_{\mathrm{T}}^{a}\right)-\Psi_{n}\left(p_{\mathrm{T}}^{t}\right)\right]\right\rangle}{\left\langle v_{n}\left(p_{\mathrm{T}}^{a}\right)^{2} v_{n}\left(p_{\mathrm{T}}^{t}\right)^{2}\right\rangle} \\
& \approx\left\langle\cos 2 n\left[\Psi_{n}\left(p_{\mathrm{T}}^{a}\right)-\Psi_{n}\left(p_{\mathrm{T}}^{t}\right)\right]\right\rangle
\end{aligned}
$$

where the last equality holds under the assumption that non-flow is approximately the same in both numerator and denominator. $C\left(\Psi_{n}^{a}, \Psi_{n}^{t}\right)$ does not depend on the flow magnitude and only has contributions from fluctuations of the flow angle $\Psi_{n}$. Values of $C\left(\Psi_{n}^{a}, \Psi_{n}^{t}\right)$ smaller than unity indicates the presence of $p_{\mathrm{T}}$-dependent flow angle fluctuations. Figure 3 shows $C\left(\Psi_{2}^{a}, \Psi_{2}^{t}\right)$ as function of $p_{\mathrm{T}}^{a}$ with $0.2<p_{\mathrm{T}}^{t}<0.6 \mathrm{GeV} / c$ in different centrality classes. $C\left(\Psi_{2}^{a}, \Psi_{2}^{t}\right)$ is consistent 


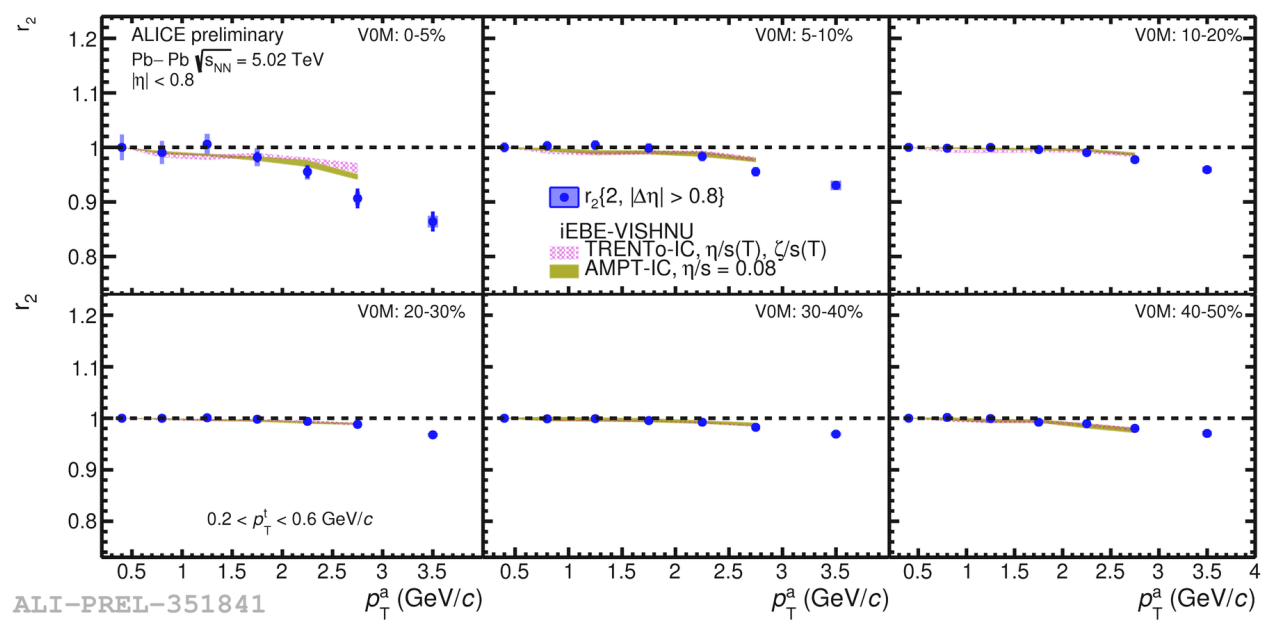

Figure 2: The factorization ratio $r_{2}$ for $\mathrm{Pb}-\mathrm{Pb}$ collisions at $\sqrt{s_{\mathrm{NN}}}=5.02 \mathrm{TeV}$. The error bars correspond to statistical uncertainties and coloured boxes correspond to systematic uncertainties. Comparison with iEBE-VISHNU hydrodynamic model with TRENTo initial conditions and temperature dependent $\eta / s(T)$ and $\zeta / s(T)$ [7], and with AMPT initial conditions and $\eta / s=0.08$ [7] are shown in coloured bands.

with unity within the uncertainties. Further study is needed to understand the contributions from fluctuations of the flow angle.

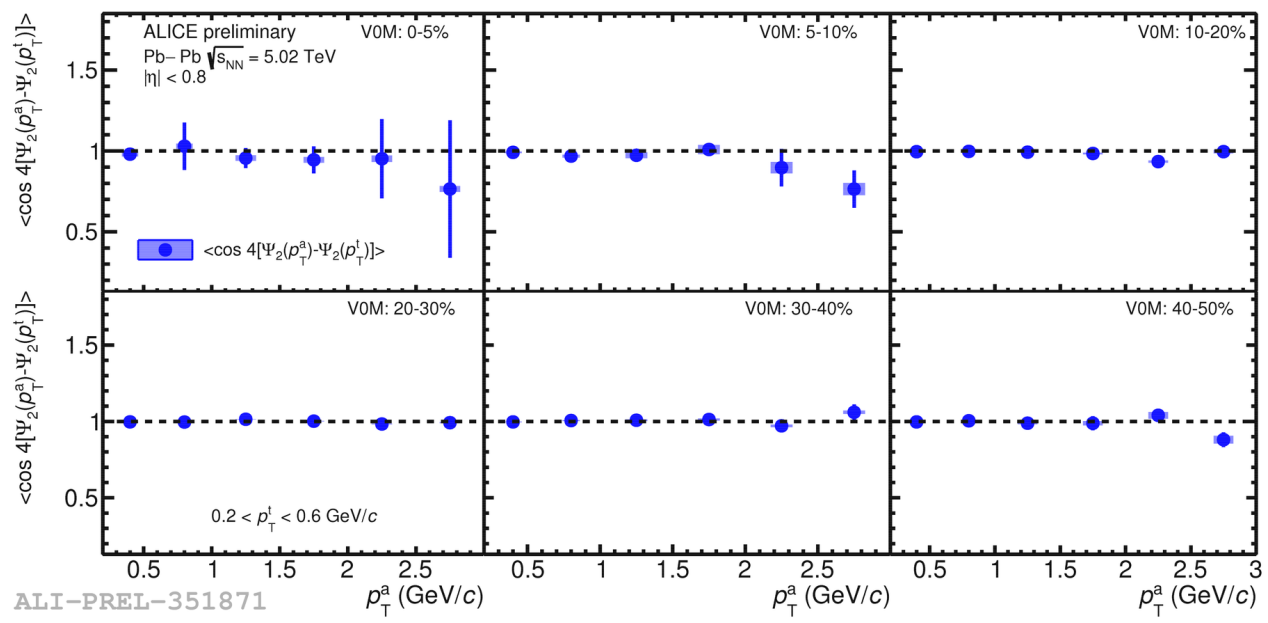

Figure 3: The double $p_{\mathrm{T}}$-differential flow angle decorrelation $C\left(\Psi_{2}^{a}, \Psi_{2}^{t}\right)$ for $\mathrm{Pb}-\mathrm{Pb}$ collisions at $\sqrt{s_{\mathrm{NN}}}=$ $5.02 \mathrm{TeV}$ for all centrality classes up to $40-50 \%$ with trigger particle $0.2<p_{\mathrm{T}}^{t}<0.6 \mathrm{GeV} / c$. The error bars correspond to statistical uncertainties and coloured boxes correspond to systematic uncertainties.

\section{Summary}

In this proceeding we present results of $v_{2}\{2\} / v_{2}$ [2] and $r_{2}$ as function of $p_{\mathrm{T}}$ in different centrality classes. The results indicate the presence of $p_{\mathrm{T}}$-dependent $V_{2}$ flow vector fluctuations in central $\mathrm{Pb}-\mathrm{Pb}$ collisions. These results can help further constrain initial conditions and the QGP 
properties. Measurement of the newly proposed observable $C\left(\Psi_{2}^{a}, \Psi_{2}^{t}\right)$ is consistent with unity within the statistical uncertainties. Further study of this observable is needed, as it can give us a new flow picture and help us understand the dynamical expansion of the created system.

\section{References}

[1] S. Voloshin and Y. Zhang. Flow study in relativistic nuclear collisions by Fourier expansion of Azimuthal particle distributions. Z. Phys., C70:665-672, 1996.

[2] U. Heinz, Z. Qiu and C. Shen. Fluctuating flow angles and anisotropic flow measurements. Phys. Rev. C 87, 034913 (2013).

[3] Gardim, Fernando G. and Grassi, Frederique and Luzum, Matthew and Ollitrault, Jean-Yves. Breaking of factorization of two-particle correlations in hydrodynamics. Phys. Rev. C 87, 031901(R) (2013).

[4] Aamodt, K. and others, ALICE collaboration, The ALICE experiment at the CERN LHC, JINST 3 S08002 (2008).

[5] A. Bilandzic, C.H. Christensen, K. Gulbrandsen, A. Hansen and Y. Zhou. Generic framework for anisotropic flow analyses with multiparticle azimuthal correlations. Phys. Rev. C 89, 064904 (2014).

[6] C. Shen, Z. Qiu, H. Song, J. Bernhard, S. Bass, and U. Heinz. The iEBE-VISHNU code package for relativistic heavy-ion collisions. Comput. Phys. Commun 199 (2016) 61:85.

[7] W. Zhao, H. Xu and H. Song. Collective flow in $2.76 \mathrm{~A} \mathrm{TeV}$ and 5.02 A TeV Pb+Pb collisions. Eur. Phys. J. C (2017) 77:645. 